\title{
The estimation of age from elastic fibers in the tunica media of the aortic wall in a thai population: a preliminary study using aorta image analysis
}

\begin{abstract}
Pornhatai Komutrattananont ${ }^{1,2}$, Patison Palee ${ }^{3}$, Sukon Prasitwattanaseree ${ }^{4,5}$, Pasuk Mahakkanukrauh ${ }^{2,5,6}$ ${ }^{1}$ PhD Degree Program in Anatomy, Department of Anatomy, Faculty of Medicine, Chiang Mai University, Chiang Mai, ${ }^{2}$ Department of Anatomy, Faculty of Medicine, Chiang Mai University, Chiang Mai, ${ }^{3}$ College of Arts Media and Technology, Chiang Mai University, Chiang Mai, ${ }^{4}$ Department of Statistics, Faculty of Science, Chiang Mai University, Chiang Mai, ${ }^{5}$ Forensic Osteology Research Center, Faculty of Medicine, Chiang Mai University, Chiang Mai, ${ }^{6}$ Excellence in Osteology Research and Training Center (ORTC), Chiang Mai University, Chiang Mai, Thailand
\end{abstract}

\begin{abstract}
Image analysis has an increasing role in the identification of individuals in forensic application. Beside the bones, microstructural of arteries can be used in age estimation study. Aorta is the largest elastic artery which consists of many elastic fibers. Elastin in arterial wall highly resist to chemical and physical influence. The purposes of the study were to quantify elastic fibers in tunica media in each location of the aorta and examine the correlation between elastic fibers and age by using image analysis program. A total of 36 human aortas were dissected in 4 locations. The aortas were obtained from cadavers with an age range of 20 to 90 years. Specimens were stained with Elastic Van Gieson staining. Histological images were investigated about elastic fibers using light microscope with cellSens program and aorta image analysis was used for the evaluation of data. The results showed that the mean percentage density of elastic fibers in the ascending aorta and the aortic arch increased. However, the mean percentage density of elastic fibers decreased in the 31 to 40 years age group in the thoracic aorta and the abdominal aorta and decreased in each location of aorta continuously until 81 to 90 years. The abdominal aorta showed the highest correlation with age $(\mathrm{r}=0.732)$ followed by the thoracic aorta, the aortic arch and the ascending aorta, respectively. Changes in the percentage density of elastic fibers in the tunica media of the aortic wall can be used to add information to age estimation for identification purposes.
\end{abstract}

Key words: Aorta, Elastic fibers, Age estimation, Image analysis, Tunica media

Received April 19, 2020; Revised May 22, 2020; Accepted June 8, 2020

\section{Introduction}

In a world in which technology is advancing rapidly, many

\author{
Corresponding author: \\ Pasuk Mahakkanukrauh (i) \\ Department of Anatomy, Faculty of Medicine \& Research Cluster in \\ Osteology Research and Training Center (ORTC), Chiang Mai University, \\ Chiang Mai 50200, Thailand \\ E-mail:pasuko34@gmail.com
}

researches have investigated the use of computer software like the image analysis tool for application in medical fields to quantify or classify the patterns of medical images. The image analysis is more important than ever since it is convenient, cost-effective, and also non-invasive method [1]. In the forensic field, identification of individuals has been done by using the technology of using images to analyze dead person such as DNA, fingerprint examination etc. It can be assessed by age, stature, gender, ethnicity which studied from bones and teeth. Age estimation is used for investigation to identify the approximate range of age of an unknown person. There 
were several studies for different organs that change with age $[2,3]$. Age is an important change of the organism in life [4]. Cell in our bodies in each stage of life develop and degenerate. There was the theory of cell changes that are correlated with age, for example collagen deposit theory, gene mutation, autoimmune theory and wear and tear theory [5]. During aging, this changes of the cell can lead to pathology of the disease [6], especially incident frequently in the cardiovascular system such as atherosclerosis which is the increase in the fatty layer. This contributes to stiffness that occurred in vessel walls [7].

From previous studies, vascular changes with age affect the internal and external structure changes [8]. With aging process, There were change of diameter size at the vascular [9]. Compositions of each tunica layers within vessel walls changed differently such as endothelial cell of tunica intima which presented irregular shape, increased collagen fibers and decreased smooth muscles cell [10]. Moreover, The elastic fibers were decreased and become fragmentation [11], especially the aorta as it is obvious. The aorta is the largest elastic artery. It is divided into 3 main locals defined by their location as follows: the ascending aorta, aortic arch, and the descending aorta. The descending aorta is divided into the thoracic aorta and abdominal aorta. On the microscopic level, the aorta consists of three layers from innermost to the outermost layer, including the tunica intima, tunica media, and tunica adventitia. Tunica intima contains a single layer of endothelial cells. The subendothelial cells contain loose connective tissue and few fibroblasts. Moreover, there is internal elastic lamina which separates tunica intima and tunica media layers. The middle layer is tunica media, which consists of many elastic fibers and is the thickest layer. It also consists of smooth muscle cell and collagen fibers. The elastic fibers arrange in concentric layer called elastic lamellae. There is also external elastic lamina which separates layers of tunica media and tunica adventitia. Tunica adventitia includes collagen fibers, some elastic fibers, fibroblasts, and mast cell. The connective tissue contains vasa vasorum which are small vessels for blood supply of the aortic wall [12-14].

In the aortic wall, Elastic fibers are associated with extension and recoil in the circulatory system. Elastin is one of the essential parts of the human tissues that provided elasticity. Moreover, elastin highly resists to chemicals hardly and physical influences [15]. Elastin behavior has related to low stiffness modulus and large extensibility because the aorta carries blood from the heart into different organs [16]. Many literatures had reported the elastic fibers related to age. Age changes of elastic fibers in tunica media of the aortic wall occurs as a change in the different locations of the aorta [17]. The increasing age causes degeneration of elastic fibers in the media and many elastin fragmentations. Ninomiya et al. [18] found that the thoracic aorta were of higher strength and elasticity than the abdominal aorta. In addition, the aorta in elderly has elasticity less than the young.

There have been few studies about the changes of elastic fibers with age in the human aorta cadaver that can improve the accuracy of investigation for the identification. Additionally, there have been no studies in Thai population by using image analysis program before. Hence, the aims of this study to quantify elastic fibers in tunica media of aortic wall in different locations with age groups by using image analysis and investigates the correlation between percentage density of elastic fibers in tunica media of the aorta.

\section{Materials and Methods}

The samples were studied in 36 human aortas (29 males and 7 females) from the autopsy of Thai cadavers were obtained from The Department of Forensic Medicine and fresh donor cadavers in the Department of Anatomy, Faculty of Medicine, Chiang Mai University. The study was done in each decade each of age ranging from 20 to 90 years old (Table 1). The criteria for exclusion were as follows: aortic aneurysms with diameter, atherosclerosis of the aorta with stage 3 (complicated lesion) in gross examination, death from infectious disease, inappropriate aortic tissue due to advanced wall decomposition, death due to burns. Each whole aorta was dissected and removed from loose connective tissues. We cut four different locations of the aorta. The ascending aorta was cut at approximately $2 \mathrm{~cm}$ above the aortic valve. The aortic arch was cut at the midpoint between

Table 1. Total number of aortic samples by age group

\begin{tabular}{cl}
\hline Age group $(\mathrm{yr})$ & Total $(\mathrm{n}=36)$ \\
\hline $20-30$ & $5(\mathrm{M}=5)$ \\
$31-40$ & $5(\mathrm{M}=5)$ \\
$41-50$ & $5(\mathrm{M}=5)$ \\
$51-60$ & $6(\mathrm{M}=4, \mathrm{~F}=2)$ \\
$61-70$ & $5(\mathrm{M}=3, \mathrm{~F}=2)$ \\
$71-80$ & $6(\mathrm{M}=4, \mathrm{~F}=2)$ \\
$81-90$ & $4(\mathrm{M}=3, \mathrm{~F}=1)$
\end{tabular}

M, male; F, female. 
the origins of the left common carotid artery and the right brachiocephalic artery. The descending thoracic aorta was cut at the midpoint between the origin of the left subclavian artery and the celiac trunk. The descending abdominal aorta was cut at a halfway between the renal arteries and inferior mesenteric artery. Each location of the aorta was sectioned circumferentially in the aortic rings and then the aortic ring was cut into rectangular strips of approximately $3 \mathrm{~mm}$ in width and $10 \mathrm{~mm}$ in length. All segments of the aorta were cut into one strip at the anterior wall. For the latter, the specimens were stored in $10 \%$ formalin for 24 hours before bringing to histological preparation. Afterwards, the specimens were prepared for histological processing and the tissue blocks were cut into a thickness of $4 \mu \mathrm{m}$ with a rotary microtome and were stained with Elastic Van Gieson staining by automatic tissue processor. Following that, the images of stained sections were visualized using a light microscope with Olympus DP73 digital microscope camera (Olympus, Tokyo, Japan). The histological images were photographed using a CellSens standard program (Olympus) and the microscopic field was analyzed using 40x magnifications. The digitization of the image was captured from the selected microscopic field. These images of a microscopic field were delivered to analyzed by using our image analysis program. The analysis program consists of four steps (Fig. 1). First, the pre-processing step aims at removing unnecessary objects and improving the quality of an image for further steps. The International Commission on Illumination color space is used to improve the quality of input images in terms of the color of histological images. Second, the segmentation step aims at selecting the region of interest (ROI). The k-nearest neighbor algorithm is applied to select elastic fibers in tunica

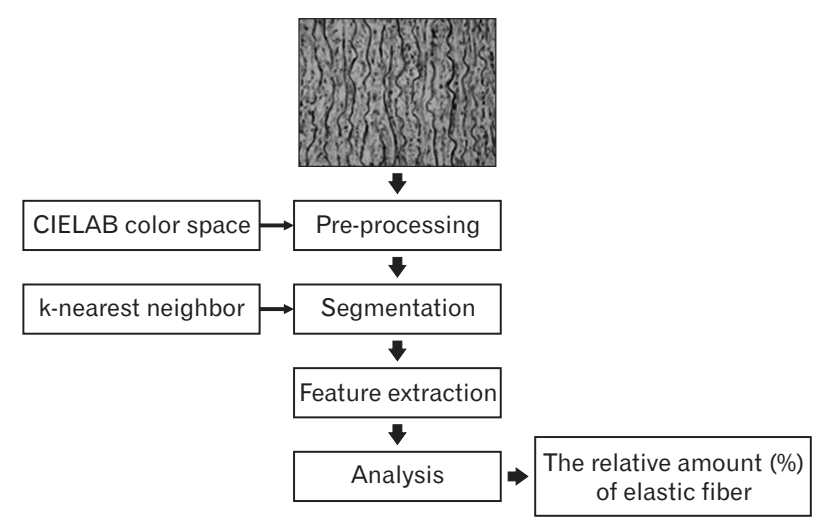

Fig. 1. Image analysis steps. CIELAB, International Commission on Illumination. media of the aorta image. The third step is feature extraction. This step aims at extracting the interesting feature of the ROI. Finally, analysis data is the last step. the analysis data was extracted for the relative amount (\%) of elastic fibers which was the ROI. The percentage density of elastic fibers in the field is calculated by the selected elastic fiber regions in the aorta image as shown in Fig. 2. All statistical analysis will be performed by using IBM SPSS Statistics for Windows, Version 19.0 (IBM Co., Armonk, NY, USA). Ethical clearance was taken by the research Ethics Committee from Faculty of Medicine, Chiang Mai University (Research ID: ANA-256105233).

\section{Results}

This study was to quantify percentage density of elastic fiber in tunica media of 36 aortas at each age group. In team of the highest percentage density in each case, it was found that the ascending aorta had the highest density followed by the aortic arch, the thoracic aorta, and the abdominal aorta, respectively. In the age group of 20 to 30 years, it was found that the arrangement of elastic lamellae was parallel, long, and continuous. The thoracic aorta had the highest of the mean percentage density of elastic fibers. The ascending aorta had the largest size of elastic lamellae. It was found thin fibers in the abdominal aorta. In age group of 31 to 40 years, the arrangement of elastic lamellae was the same as the previous age group. It was found that the mean percent-

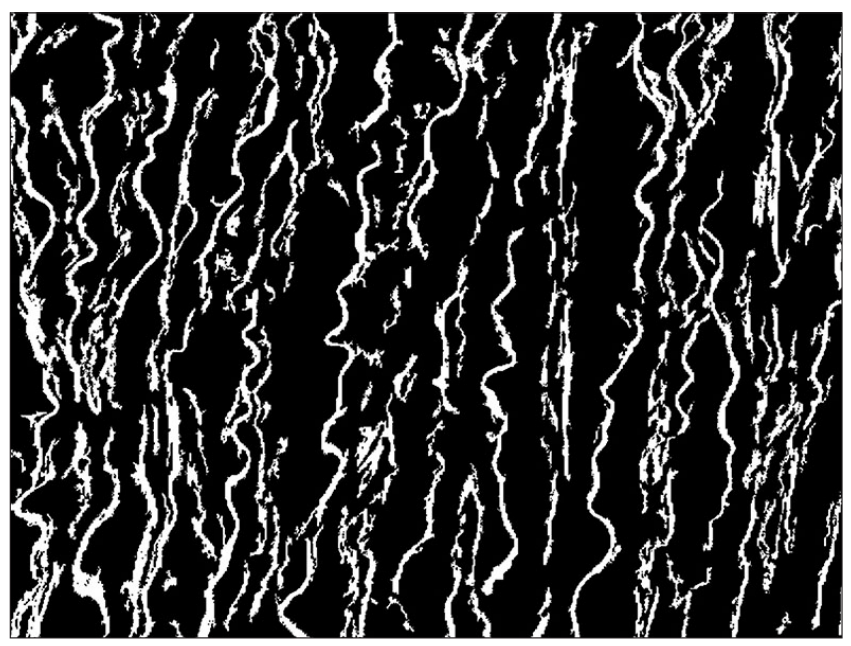

Fig. 2. Demonstration of the analyzed picture of the aortic segment using Aorta Image Analysis program. Percentage density of elastic fibers in tunica media of the aorta. White color, elastic fibers (region of interest); Black color, sections of no-interest. 
age density of elastic fibers in the ascending aorta and the aortic arch increased while the mean percentage density of elastic fibers in the thoracic aorta and the abdominal aorta decreased. The age group of 41 to 50 years had a decrease of the mean percentage density of elastic fibers in each location of the aorta. Moreover, the arrangement of elastic fibers began to slightly separate from elastic lamellae in each location of the aorta. In age group of 51 to 60 years, It was found that the mean percentage density of elastic fibers in the ascending aorta and the aortic arch had a value that was close to the previous group, but the mean percentage density of elastic fibers in the thoracic aorta and the abdominal aorta were found decreased. The arrangement of elastic fibers was clearly separate in each location. In addition, they were thinner and smaller especially in the thoracic aorta and the abdominal aorta. In age group of 61 to 70 years, it was found that the ascending aorta, the aortic arch, and the thoracic aorta had a slightly difference of the mean percentage density of elastic fibers and they had the similar sharp of elastic lamellae to the previous group. The abdominal aorta had a decrease of the mean percentage density of elastic fibers and it was the thinnest and smallest size of elastic lamellae. The mean percentage density of elastic fibers in the age range of 71 to 80 years decreased in each location of the aorta. The elastic
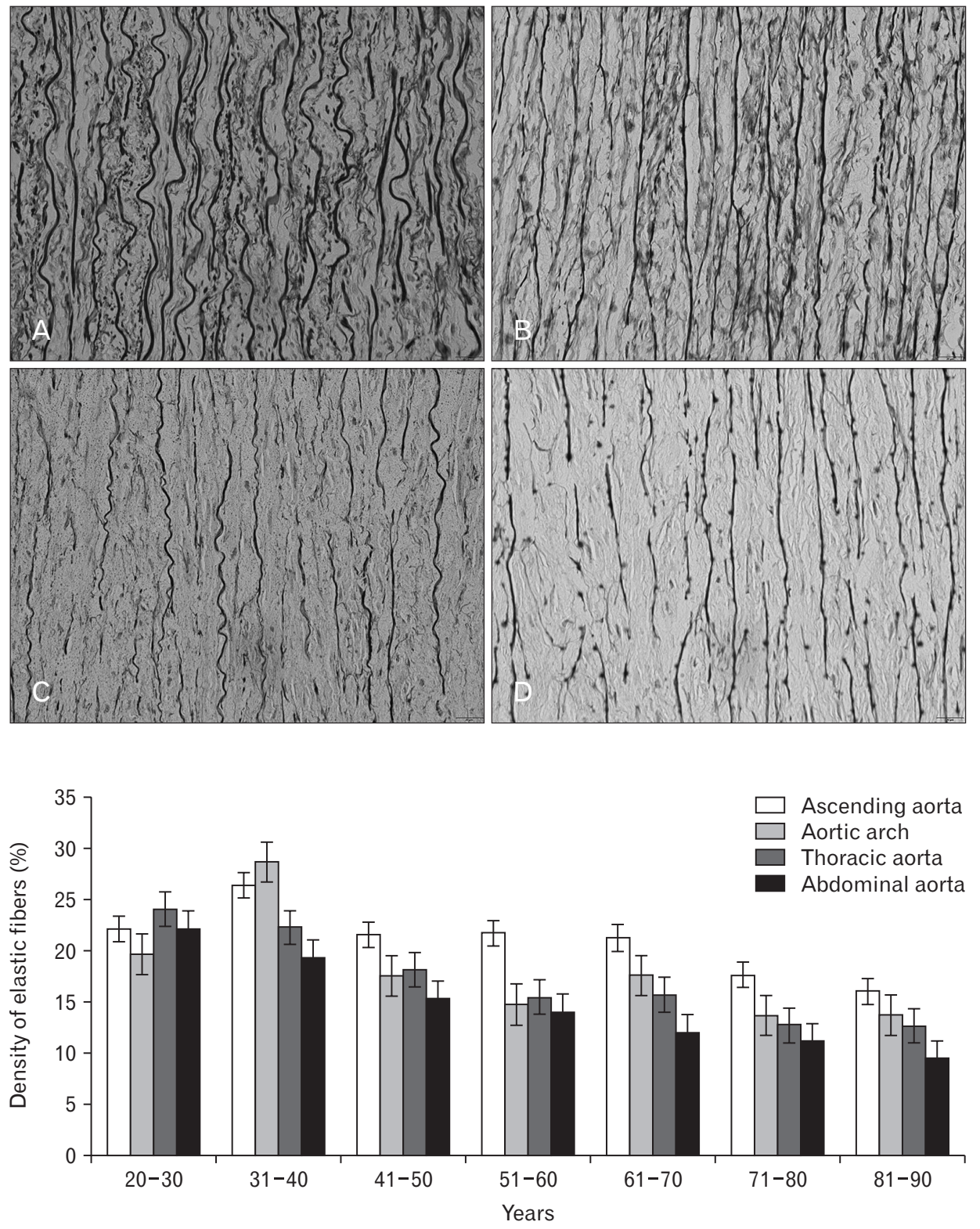

Fig. 3. Example of elastic fibers in tunica media from the abdominal aorta at different ages (Elastic Van Gieson, $\times 40$ ). (A) A 20 years of human aorta, (B) 43 years of human aorta, (C) 61 years of human aorta, (D) 81 years of human aorta. Scale Bars $=20 \mu \mathrm{m}(\mathrm{A}-\mathrm{D})$.

Fig. 4. The bar chart showing mean percentage density of elastic fibers in various age groups. 
A
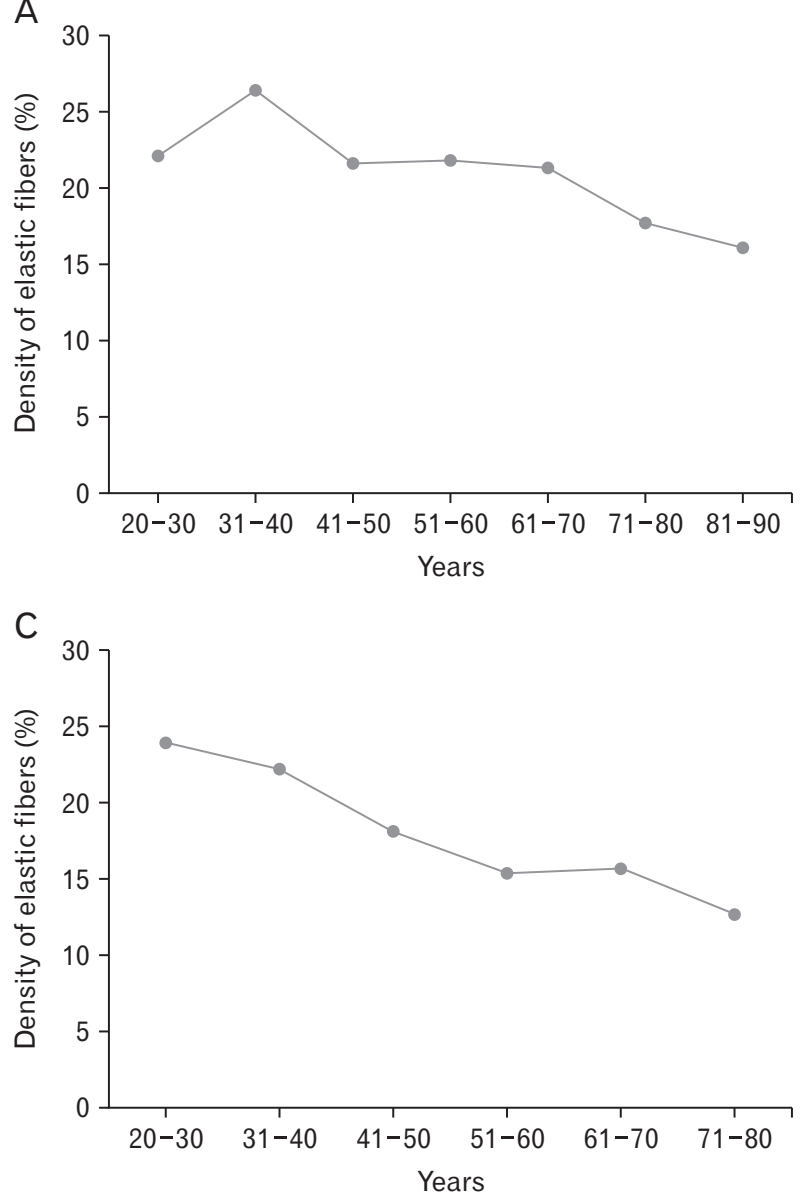

B

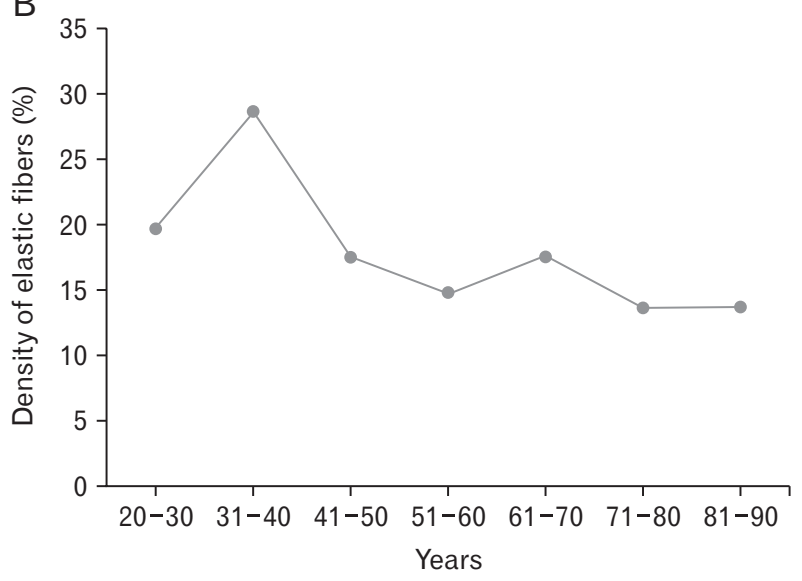

D

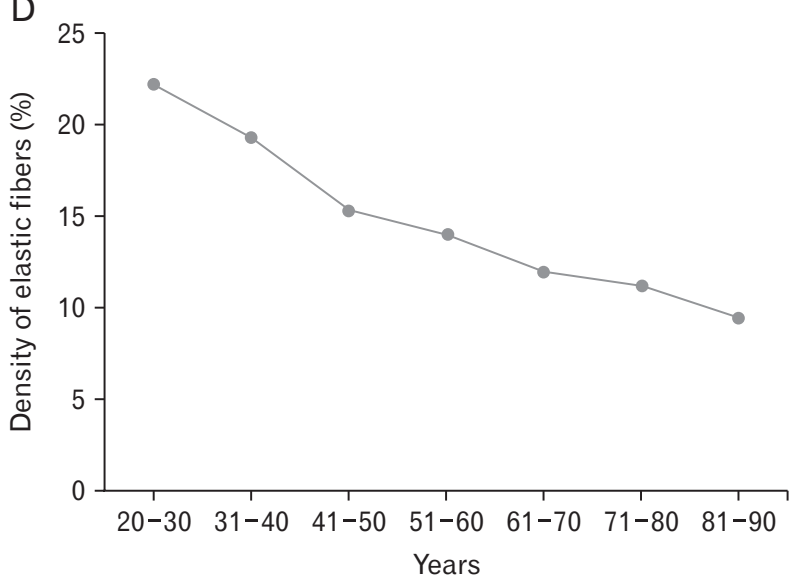

Fig. 5. The scatter plot showing mean percentage density of elastic fibers in various age groups with different locations of the aorta. (A) Ascending aorta, (B) aortic arch, (C) thoracic aorta, (D) abdominal aorta.

lamellae of the thoracic aorta and the abdominal aorta were more separated than the ascending aorta and the aortic arch clearly, and they had thin and small size of elastic lamellae. In age range of 81 to 90 years, it was found that the mean percentage density of elastic fibers decreased in the ascending aorta and the abdominal aorta, but the thoracic aorta and the aortic arch were similar to the previous group. The arrangements of elastic lamellae of the ascending aorta and the aortic arch still appeared clearly, but the thoracic aorta and the abdominal aorta were thin in size and separated in parallel line. Fig. 3 showed elastic fibers changes in tunica media from the abdominal aorta in different ages. The Mean values for the percentage density of elastic fibers in tunica media in different locations of the aorta were demonstrated in Figs. 4 and 5.

There was a statistically significant negative correlation between each age group and percentage density of elastic fibers of tunica media in each location of the aorta. The results

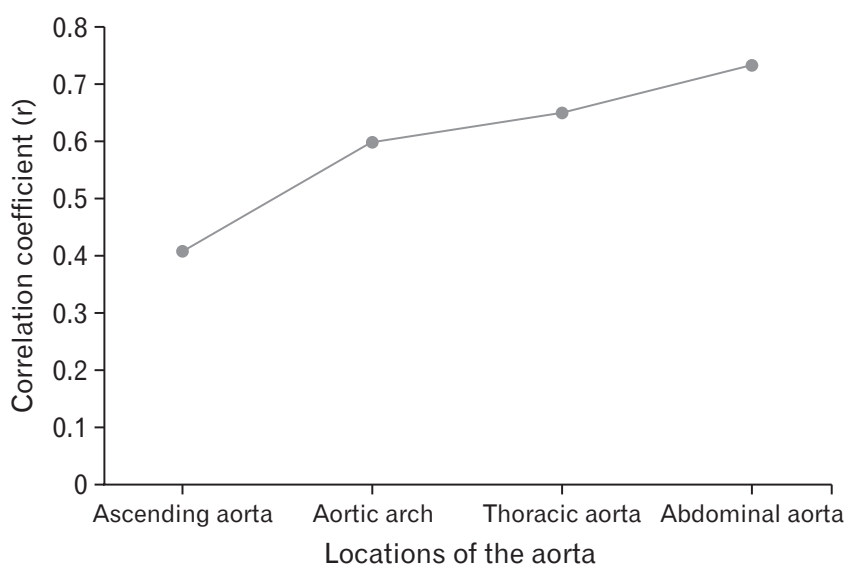

Fig. 6. The scatter plot showing the correlation coefficient with age in different locations of the aorta. 
showed that the percentage density of elastic fibers of tunica media in each location of the aorta tended to decrease with advancing age. The scatter plot in Fig. 6 demonstrate the correlation with age in each location of the aorta. A significant correlation was found with age at 0.732 in the abdominal aorta and this was highest correlation when compared to other locations. The thoracic aorta correlated significantly with age at 0.648 and the aortic arch significantly correlated with age at 0.598 . The ascending aorta correlated significantly with age at 0.408 .

\section{Discussion}

From previous studies, there were different various methods for studying the quality and quantity of elastic fibers such as Biochemical detection methods, electron microscope and light microscope. Elastic fibers contained elastic filament and microfibrils. Its function is accumulating energy and driving in the recoil [19]. The elastic fibers have characteristics that resemble rubber that causes the object to stretch, contract and resilience. Elastic fibers can change according age, disease and strong chemicals. There are split and separate of elastic fibers with the increasing of age. Decreasing of elastic fibers causes reduced flexibility $[20,21]$. Elastic fibers are a structure of lung, skin, elastic artery ligament and tendon. Mario Luiz Quintas et al. [22] showed that the elastic fibers of the interfoveolar ligament in young men distributed uniformly and were parallel with collagen, conversely in the elderly men, there were thickened and fragmented of elastic fibers. In addition, Frances et al. [23] found the area of mature elastic fibers in the reticular dermis by computerized digital image analysis. The elastic fibers increased with age after sixth decade. With aging, loss of elastic fibers caused the loss of skin thickness [23]. Another study showed increasing of the surface density in elastic fibers at the superficial and deep dermis with age [24]. Age was involved with changes of vascular system such as diameter, thickness, and components within aortic wall [25]. Hosoda et al. [26] investigated elastic fibers of the aorta and pulmonary artery in Japanese and found that youths have a higher of elastic content in the aorta than older age. With increasing age, elastic content in the aorta tended to decrease. The change of elastic content in the pulmonary artery was less than in the aorta which may be due to the effect of blood pressure on different hemodialysis [26]. Tunica media in the aorta consisted of collagen, elastic fiber, smooth muscle cells, and mucopolysaccharide. Elastic fibers arranged in lamellae from throughout the length of the aorta, but the quantity and shape were different $[27,28]$. Changing of the elastic structure in the aorta arranged dispersedly and broken with age $[29,30]$. A recent study of elastic fibers in the aorta found that the percentage of elastin of area fraction in the ascending aorta in the elderly (52-85 years) was a negative tendency and the correlation between age was -0.66 [31].

Additionally, it was found that there was more parallel elastin arrangement in 20-year-old group and the number of elastin were increased with age. After that, it remained constant at the age of 50 and decreased at the age range of 70 to 80 years [32-34]. Changes in the characteristics of elastic fibers were mostly found in 50-years age group [35]. Schlatmann and Becker [27] revealed that the thoracic aorta was greater elastic fibers than the abdominal aorta and the abdominal aorta was split more than the thoracic aorta [36]. According to Wolinsky and Glagov [37] study, they have showed that the abdominal aorta was more involved with atherosclerosis than the thoracic aorta and the number of elastic fibers in media decreased with distance from aortic root. Atherosclerosis occurred after 40 years of age. It was found that elastic content significantly decreased in the infrarenal aorta and atherosclerosis often occur with the distal portion of the aorta. The results were consistent with this study, we found that atherosclerosis at the abdominal aorta was greater than other areas, followed by the aortic arch, thoracic aorta, and ascending aorta, respectively. It was revealed that the mean percentage of elastic density of the ascending aorta and aortic arch at 51 to 70 years still remained and decreased at the age of 71 to 80 . Then, at the age of 81 to 90 , it decreased again in the ascending aorta. Conversely, the aortic arch remained constant. The thoracic aorta decreased in mean value percentage elastic density at 51 to 60 years and was remained at 61 to 70 years. Then, it decreased at 71 to 80 years. After that, the percentage elastic density was constant. The elastic fibers in the abdominal aorta decreased at the age above 50 years. Age is associated with elastic fragment [30]. The principle of wear and tear regarding blood pressure in multiple directions was affected to elastic lamellae fragment in tunica media [38]. It was separated initially at the age 30 $[39,40]$. Similar to the ascending aorta, the aortic arch and proximal portion of the thoracic aorta are responsible for receiving high pressure blood flow and blood transports to the organs and tissues, causing the aortic in each part to have different elastic characteristics. As a result, each loca- 
tion of the aorta has a different elastic characteristic $[29,41$, 42]. Decreasing and separation of elastic fibers affected the decreased elasticity of the blood vessels that caused the blood vessels, To be more expanded, with increased collagen [40, $43,44]$. Therefore, it caused stenosis and affected blood flow that led to high blood pressure and atherosclerosis [40, 42].

This study is a new preliminary study about elastic fibers in the normal aorta in Thai population. The limitation of this study was the number of samples in each group with both sexes. The number of samples in this study were selected from autopsy and fresh donor cadavers without aortic diseases and trauma. Therefore, this resulted in receiving a few numbers of samples, especially for the young females from autopsy. In future study, a larger sample group will be evaluated and increased for more precise database. In addition, we will add parameters with age in the aorta such as collagen and thickness of each layer in the aortic wall. Another future study will develop continuously the aorta image analysis program of progressive approach basis for estimating age from the elastic fibers in order to be more accurate, efficiency and reduce errors. We will establish an effective equation for age estimation. From our study, the aorta image analysis program was created, percentage density of elastic fibers in tunica media of each location of the aorta was also important as this information may be used for age investigation. We expect that it will be easily accessible to use and can be applied to the application for age estimation that reduces time to determine the unknown person in the forensic identification.

In conclusion, the presented results demonstrate application of aorta image analysis program using elastic fibers in tunica media of the aorta. There were negative correlations between age and percentage density of elastic fibers within aortic wall in each location. The abdominal aorta showed the highest correlation with age and the ascending aorta showed the least correlation. Since each location of the aorta works at a different blood pressure which is affected by the distribution and function. These changes caused reduce elasticity within the aortic wall. However, we should have methods that can increase accuracy, easier and more convenient to use. According to our study, image analysis program to be used as a database of percentage density of elastic fibers with age groups for the identification of individuals in forensic field.

\section{ORCID}

Pornhatai Komutrattananont: https://orcid.org/0000-0001-5347-6908

Patison Palee: https://orcid.org/0000-0003-4538-4266

Sukon Prasitwattanaseree:

https://orcid.org/0000-0002-1987-773X

Pasuk Mahakkanukrauh:

https://orcid.org/0000-0003-0611-7552

\section{Author Contributions}

Conceptualization: PK, PP, PM. Data acquisition: PK. Data analysis or interpretation: PK, SP. Drafting of the manuscript: PK, PP, PM. Critical revision of the manuscript: PK, PP, SP, PM. Approval of the final version of the manuscript: all authors.

\section{Conflicts of Interest}

No potential conflict of interest relevant to this article was reported.

\section{Acknowledgements}

This study was funded by the Faculty of Medicine, Chiang Mai University, Chiang Mai, Thailand (Research ID: ANA2561-05233).

\section{References}

1. Prats-Montalbán JM, de Juan A, Ferrer A. Multivariate image analysis: a review with applications. Chemometr Intell Lab Syst 2011;107:1-23.

2. Ritz-Timme S, Cattaneo C, Collins MJ, Waite ER, Schütz HW, Kaatsch HJ, Borrman HI. Age estimation: the state of the art in relation to the specific demands of forensic practise. Int J Legal Med 2000;113:129-36.

3. Lynnerup N. Forensic anthropology and human identification. Scandinavian J Forensic Sci 2013;19:16-38.

4. Webb RC, Inscho EW. Age-related changes in the cardiovascular system. In: Michael PL, editor. Hypertension in the elderly. Totowa: Humana Press; 2005. p.11-21.

5. Gupta SD, Gupta SK, Pal DK, Sarawagi R, Gupta P. Microscopic study of aorta in relation of different age groups: an observational study. Int J Biol Med Res 2011;2:398-403.

6. Lakatta EG, Levy D. Arterial and cardiac aging: major shareholders in cardiovascular disease enterprises: part II: the aging heart in health: links to heart disease. Circulation 2003;107:346-54.

7. Collins JA, Munoz JV, Patel TR, Loukas M, Tubbs RS. The anatomy of the aging aorta. Clin Anat 2014;27:463-6. 
8. Izzo JL Jr, Shykoff BE. Arterial stiffness: clinical relevance, measurement, and treatment. Rev Cardiovasc Med 2001;2:29-40.

9. Xu X, Wang B, Ren C, Hu J, Greenberg DA, Chen T, Xie L, Jin $\mathrm{K}$. Age-related impairment of vascular structure and functions. Aging Dis 2017;8:590-610.

10. Cheuk BL, Cheng SW. Expression of integrin alpha5beta1 and the relationship to collagen and elastin content in human suprarenal and infrarenal aortas. Vasc Endovascular Surg 2005;39:245-51.

11. Samila ZJ, Carter SA. The effect of age on the unfolding of elastin lamellae and collagen fibers with stretch in human carotid arteries. Can J Physiol Pharmacol 1981;59:1050-7.

12. Komutrattananont P, Mahakkanukrauh P, Das S. Morphology of the human aorta and age-related changes: anatomical facts. Anat Cell Biol 2019;52:109-14.

13. Gartner LP, Hiatt JL. Color atlas and text of histology. Philadelphia: Wolters Kluwer Health/Lippincott Williams \& Wilkins; 2014.

14. Mitchell JRA, Schwartz CJ, Pickering G. Arterial disease. Oxford: Blackwell Scientific Publications; 1965.

15. Berillis $P$. The role of collagen in the aorta's structure. Open Circ Vasc J 2013;6:1-8.

16. Emmott A, Garcia J, Chung J, Lachapelle K, El-Hamamsy I, Mongrain R, Cartier R, Leask RL. Biomechanics of the ascending thoracic aorta: a clinical perspective on engineering data. Can J Cardiol 2016;32:35-47.

17. Tsamis A, Krawiec JT, Vorp DA. Elastin and collagen fibre microstructure of the human aorta in ageing and disease: a review. J R Soc Interface 2013;10:20121004.

18. Ninomiya OH, Tavares Monteiro JA, Higuchi Mde L, PuechLeão P, de Luccia N, Raghavan ML, da Silva ES. Biomechanical properties and microstructural analysis of the human nonaneurysmal aorta as a function of age, gender and location: an autopsy study. J Vasc Res 2015;52:257-64.

19. Sherratt MJ. Tissue elasticity and the ageing elastic fibre. Age (Dordr) 2009;31:305-25.

20. Schofield JD, Weightman B. New knowledge of connective tissue ageing. J Clin Pathol Suppl (R Coll Pathol) 1978;12:174-90.

21. Gosline JM. The elastic properties of rubber-like proteins and highly extensible tissues. Symp Soc Exp Biol 1980;34:332-57.

22. Quintas ML, Rodrigues CJ, Yoo JH, Rodrigues Junior AJ. Age related changes in the elastic fiber system of the interfoveolar ligament. Rev Hosp Clin Fac Med Sao Paulo 2000;55:83-6.

23. Frances C, Branchet MC, Boisnic S, Lesty CL, Robert L. Elastic fibers in normal human skin. Variations with age: a morphometric analysis. Arch Gerontol Geriatr 1990;10:57-67.

24. Robert C, Lesty C, Robert AM. Ageing of the skin: study of elastic fiber network modifications by computerized image analysis. Gerontology 1988;34:291-6.

25. Toda T, Tsuda N, Nishimori I, Leszczynski DE, Kummerow FA. Morphometrical analysis of the aging process in human arteries and aorta. Acta Anat (Basel) 1980;106:35-44.

26. Hosoda Y, Minoshima I. Elastin content of the aorta and the pulmonary artery in the Japanese. Angiology 1965;16:325-32.
27. Schlatmann TJ, Becker AE. Histologic changes in the normal aging aorta: implications for dissecting aortic aneurysm. Am J Cardiol 1977;39:13-20.

28. Fritze O, Romero B, Schleicher M, Jacob MP, Oh DY, Starcher B, Schenke-Layland K, Bujan J, Stock UA. Age-related changes in the elastic tissue of the human aorta. J Vasc Res 2012;49:77-86.

29. Greenwald SE. Ageing of the conduit arteries. J Pathol 2007;211:157-72.

30. Robert L, Robert AM, Fülöp T. Rapid increase in human life expectancy: will it soon be limited by the aging of elastin? Biogerontology 2008;9:119-33.

31. Yamada H, Sakata N, Wada H, Tashiro T, Tayama E. Agerelated distensibility and histology of the ascending aorta in elderly patients with acute aortic dissection. J Biomech 2015;48:3267-73.

32. Myers VC, Lang WW. Some chemical changes in the human thoracic aorta accompanying the aging process. J Gerontol 1946;1(Pt 1 4):441-4.

33. Faber M, Møller-Hou G. The human aorta. V. Collagen and elastin in the normal and hypertensive aorta. Acta Pathol Microbiol Scand 1952;31:377-82.

34. Alex RB, Amma LKK. Microanatomical study of age changes in tunica media of ascending aorta. J Evol Med Dent Sci 2016;5:7409-12.

35. Foster LS. Changes occurring in the elastic fibers of the aorta with advancing Age. J Med Res 1909;21:297-311.

36. Hosoda Y, Kawano K, Yamasawa F, Ishii T, Shibata T, Inayama S. Age-dependent changes of collagen and elastin content in human aorta and pulmonary artery. Angiology 1984;35:615-21.

37. Wolinsky H, Glagov S. Comparison of abdominal and thoracic aortic medial structure in mammals. Deviation of man from the usual pattern. Circ Res 1969;25:677-86.

38. Osler W. The principles and practice of medicine: designed for the use of practitioners and students of medicine. New York: D. Appleton and Company; 1892.p. 278.

39. Nichols WW, O’Rourke MF, Vlachopoulos C, Hoeck AP, Reneman RS. McDonald's blood flow in arteries: theoretic, experimental, and clinical principles. 6th ed. London: Hodder Arnold; 2011.

40. O'Rourke MF, Hashimoto J. Mechanical factors in arterial aging: a clinical perspective. J Am Coll Cardiol 2007;50:1-13.

41. O'Rourke MF, Holloway C, O'Rourke J. The proximal thoracic aorta: keystone or Achilles' heel? J Am Coll Cardiol 2014;64:2630-2.

42. Halloran BG, Davis VA, McManus BM, Lynch TG, Baxter BT. Localization of aortic disease is associated with intrinsic differences in aortic structure. J Surg Res 1995;59:17-22.

43. Haskett D, Johnson G, Zhou A, Utzinger U, Vande Geest J. Microstructural and biomechanical alterations of the human aorta as a function of age and location. Biomech Model Mechanobiol 2010;9:725-36.

44. Avolio A, Jones D, Tafazzoli-Shadpour M. Quantification of alterations in structure and function of elastin in the arterial media. Hypertension 1998;32:170-5. 